\title{
Star Formation Close to Sgr A* and Beyond the Nuclear Cluster
}

\author{
Farhad Yusef-Zadeh ${ }^{1}$ and Mark Wardle ${ }^{2}$ \\ ${ }^{1}$ CIERA, Department of Physics and Astronomy, Northwestern University, \\ 2145 Sheridan Rd, Evanston, IL 60208, US \\ email: zadeh@northwestern.edu \\ ${ }^{2}$ Department of Physics and Astronomy and Research Center for Astronomy, \\ Astrophysics \& Astrophotonics, Macquarie University, Sydney NSW 2109, Australia \\ email: mark. wardle@mq.edu. au
}

\begin{abstract}
Two modes of star formation are involved to explain the origin of young stars near Sgr A*. One is a disk-based mode, which explains the disk of stars orbiting Sgr A*. The other is the standard cloud-based mode observed in the Galactic disk. We discuss each of these modes of star formation and apply these ideas to the inner few parsecs of Sgr A*. In particular, we focus on the latter mode in more detail. We also discuss how the tidal force exerted by the nuclear cluster makes the Roche density approaching zero and contributes to the collapse of molecular clouds located tens of parsecs away from Sgr A*.
\end{abstract}

Keywords. Galaxy: nucleus, stars: formation, ISM: clouds

\section{Introduction}

The environment of Sgr A*, the 4 million Solar mass black hole at the center of the Galaxy, provides a window to close-up study of the center of galaxies and is a potential Rosetta Stone for understanding star formation under extreme physical conditions. A critical question is whether tidal shear in the vicinity of supermassive black holes (SMBHs) is able to completely suppress or induce star formation.

Star formation is modified by tidal effects due to Sgr $A^{*}$ as well as high external pressure due to variety of processes close to Sgr A*. There is empirical evidence for three different regimes of star formation in this region of the Galaxy, as described below.

First, close to Sgr A*, there is a concentration of over 100 OB stars within 0.04 and 0.4 pc of Sgr A* providing strong evidence for formation of stars within $0.5 \mathrm{pc}$ of Sgr $\mathrm{A}^{*}$ in the last few million years (Paumard et al. 2006; Lu et al. 2009). To explain star formation in a disk, a plausible suggestion is that gravitational collapse took place in a gaseous disk captured by the black hole. Star formation in a captured disk around Sgr A* explains the compactness of the observed stellar disk. This implies that the raw material for the disk was captured from a cloud as it temporarily engulfed Sgr A* several million years ago while passing through the central parsec of the Galaxy. Due to the cancellation of angular momentum of the captured cloud material that passes on opposite sides of the black hole, it naturally produces a compact, gravitationally unstable disk (Wardle and Yusef-Zadeh 2008, 2014). One of the key feature of this mechanism is that clouds of gas can be brought in very close to supermassive black holes. This is mainly because of the loss of angular momentum of the initial cloud, thus the captured cloud generates not only a high rate of star formation but also provides the fuel necessary to feed SMBHs.

Second, massive stars such as IRS 8 and about ten bow-shock early type stars are distributed within the ionized streamers 0.5-2 pc from Sgr A* (Geballe et al. 2006; Sanchez- 


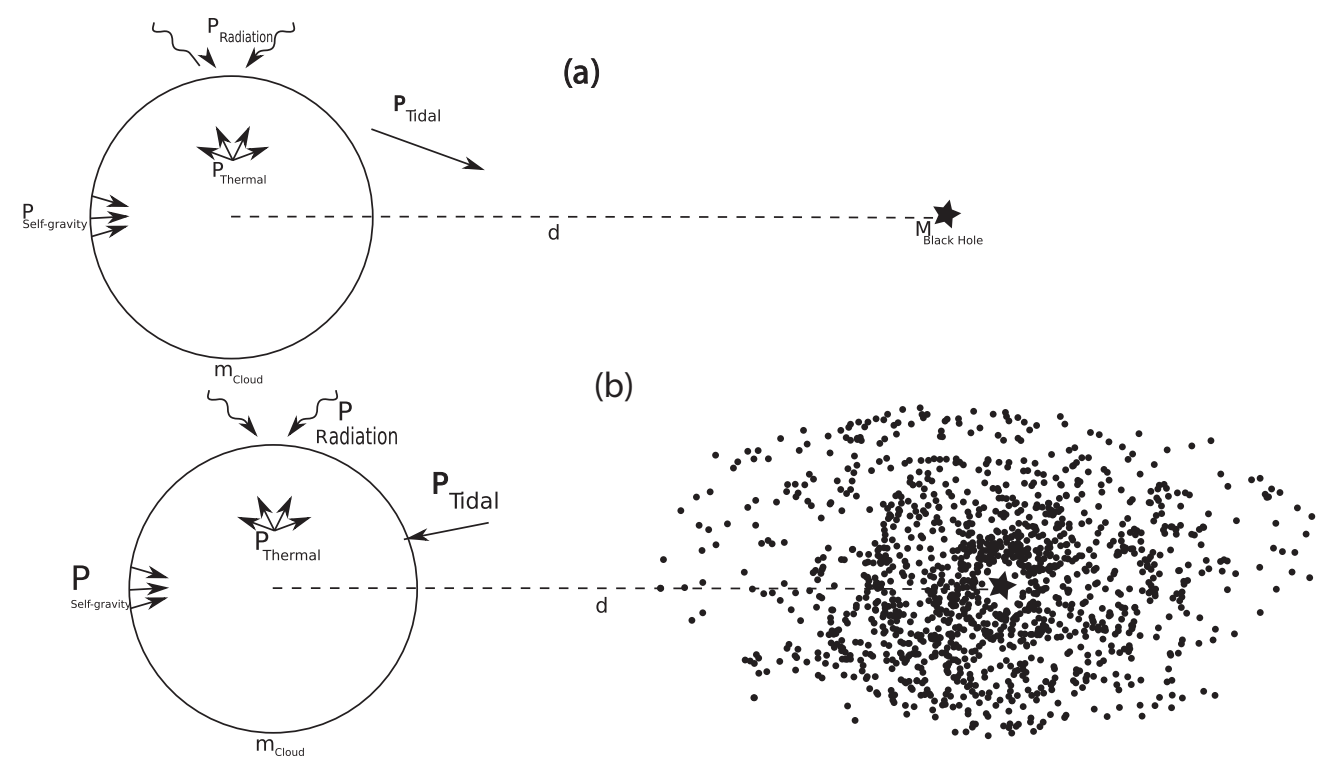

Figure 1. (a) Top A Schematic diagram showing the four forces that a cloud experiences as it approaches Sgr A* with its point mass gravitational potential. (b) Bottom Same as (a) except that an extended concentration of mass (the nuclear cluster) serves as the gravitational potential and the tidal force on the cloud becomes compressive.

Bermudez et al. 2014). On a scale of tens of parsecs, there are also clusters of massive young stars (e.g., the Arches and Quintuplet star clusters) with similar ages to those of the central cluster near Sgr A* (Stolte et al. 2015). The question is how star formation proceeds in these three different regions.

In addition to the population of young massive stars near Sgr A*, several lines of evidence point to on-going star formation within two parsecs of Sgr A*. First, the discovery of water masers with multiple and single velocity components (Yusef-Zadeh et al. 2015a). Second, SED modeling of 64 infrared excess sources in the inner pc of Sgr A* are consistent with their being YSOs (Yusef-Zadeh et al. 2015a). Third, 44 partially resolved compact sources with size scales ranging between 400 and 1600 AUs have been identified (Yusef-Zadeh et al. 2015b). The bow-shock appearance of these sources facing the direction of Sgr A* suggests a population of photoevaporative protoplanetary disks (proplyds) associated with newly formed low mass stars. The disks are externally illuminated by strong Lyman continuum radiation from the $100 \mathrm{OB}$ and WR massive stars distributed within $0.5 \mathrm{pc}$ of $\mathrm{Sgr} \mathrm{A}^{*}$. We have recently detected millimeter emission from five proplyd candidates supporting the idea that these sources have cool disks (YusefZadeh et al. 2016; submitted). Fourth, several SiO (5-4) sources within a pc of Sgr A* are suggestive of YSOs with protostellar outflows. Their SiO (5-4) luminosities and their velocity widths are similar to protostellar outflows in star forming clouds in the disk of the Galaxy (Yusef-Zadeh et al. 2013). Finally, a recent ALMA study of this region (Tsuboi et al. 2016) suggests molecular clumps of 10-100 Solar mass, distributed in the streamers. Altogether the evidence for on-going star formation implies that stellar disk of $\mathrm{OB}$ stars is formed close to Sgr $\mathrm{A}^{*}$ rather than being migrated from large distances. Here, we focus on the mode of star formation beyond the inner $0.5 \mathrm{pc}$ of Sgr $\mathrm{A}^{*}$ and summarize the results of a recent work, details of which can be found in Wardle and Yusef-Zadeh (2016, in preparation). 


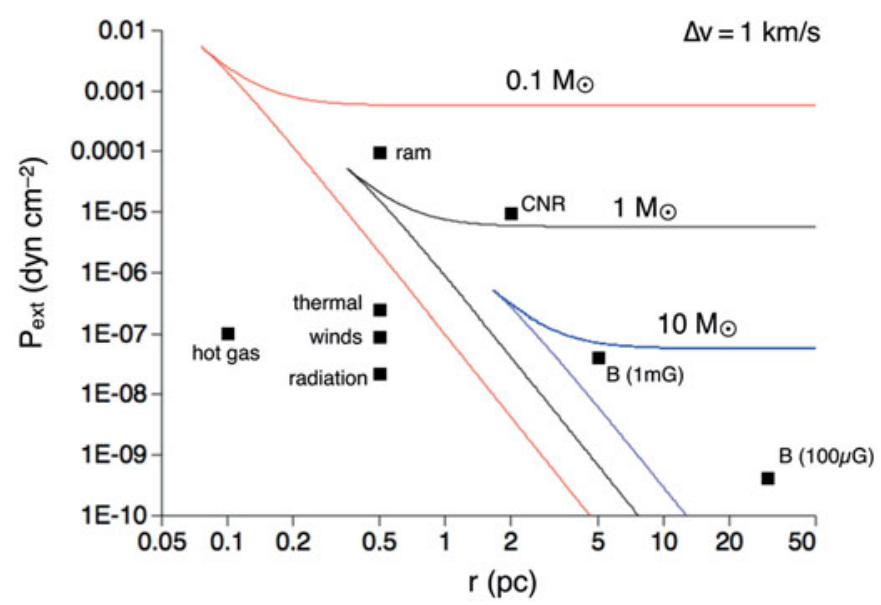

Figure 2. The external pressure experienced by a Galactic center cloud as a function of distance. The plots in the top, middle and bottom correspond to 0.1, 1 and 10 Solar mass cloud masses, respectively.

\section{Cloud-based Mode of Star Formation}

Although cloud capture model can explain the origin of the stellar disk orbiting Sgr A*, a different process is needed to explain the formation of isolated stars or of clusters beyond $0.5 \mathrm{pc}$ of Sgr $\mathrm{A}^{*}$. The gravitational potential within the inner $\sim 5 \mathrm{pc}$ is dominated by the mass of Sgr A*. The Roche density is $10^{8} \mathrm{~cm}^{-3}$ at $1 \mathrm{pc}$ from $\mathrm{Sgr} \mathrm{A}^{*}$ and the gas density inferred from a number of measurements is lower by at least 1.5 orders of magnitude. However, the gravitational stability of a cloud also depends on the internal and external pressures (Chen et al. 2016; Wardle and Yusef-Zadeh 2016). Figure 1a demonstrates the disruptive forces due to the tidal force from $\mathrm{Sgr} \mathrm{A}^{*}$ and the expansive internal pressure. These are opposed by confining forces, self-gravity and compression by external pressure such as radiation, cosmic rays and shocks. Thus, the stability and the evolution of a cloud clearly depends on the balance between the internal and external forces.

Figure 2 shows the range of external pressures for which clouds with $1 \mathrm{~km} \mathrm{~s}^{-1}$ line width are stable as a function of distance from Sgr $A^{*}$. There are different types of pressure forces acting on a cloud at different distance from Sgr A*. The calculated value of pressure on each curve should be compared to the actual pressure at each radius, which is contributed by a variety of different mechanisms such as the ram pressure associated with motion through the external medium or the pressure exerted by a jet or outflow from Sgr $A^{*}$ that impinges upon the cloud. The circumnuclear molecular ring with its high gas density, the hot X-ray gas, stellar winds from massive stars, the UV radiation from massive stars, the ionized thermal gas in the mini-spiral (the streamers) and the magnetic field contribute within a few parsecs of Sgr A*.

By way of illustration consider the fate of a one Solar mass cloud. Figure 2 shows that this cloud could collapse if it is at least $0.5 \mathrm{pc}$ from $\mathrm{Sgr} \mathrm{A*}$. If the cloud is closer than this, it will be torn apart by tidal effects. In the region between the diagonal and horizontal boundaries, the cloud remains stable and neither collapses nor is disrupted. One of the important implications is that lower mass clouds can approach Sgr A* more closely than high mass clouds. Given sufficient external pressure, lower mass stars are preferentially formed closer to the black hole. This implies that a population of proplyds associated with newly formed low mass stars should be in the vicinity of Sgr A* (see Yusef-Zadeh et al. 2015b). 


\section{Star Formation Beyond the Nuclear Cluster}

SMBHs in external galaxies with a mass $\sim 100-1000$ times Sgr A*, dominate the nuclear stellar potential within $100 \mathrm{pc}$ to $1 \mathrm{kpc}$. Star formation in the innermost regions of galaxies is generally presumed to be suppressed by strong tidal forces. In the case of $\operatorname{Sgr} \mathrm{A}^{*}$, the potential on 10-200 pc scales is dominated by the nuclear cluster. It is generally presumed (e.g. Morris \& Serabyn 1996) that tidal forces can only be overcome if the gas density exceeds the Roche density based on the interior mass. However, this condition may never arise since the Roche density drops to zero wherever the gravitational potential is not Keplerian, as described below.

It turns out that tidal forces respond differently when a cloud is experiencing a potential dominated by a point mass, Sgr A*, vs an extended mass concentration (e.g., the nuclear cluster (Jog 2013; Wardle \& Yusef-Zadeh 2016). The schematic diagram in Figure 1b presents the distribution of the nuclear cluster centered on $\mathrm{Sgr} \mathrm{A}^{*}$. A cloud within a couple of parsecs experiences mainly the Keplerian potential of Sgr $\mathrm{A}^{*}$ and at large distances beyond a few parsecs, the potential of the nuclear cluster becomes significant. The rotation curve is Keplerian in the inner parsec, where the enclosed mass is dominated by Sgr $\mathrm{A}^{*}$, flattens between 1 and $10 \mathrm{pc}$ as the stellar cluster starts to dominate the enclosed mass, and slowly rises beyond that. The consequence of such a change in the mass distribution profile is that clouds latteral compression by the tidal force of the nuclear cluster dominates radial stretching. Another consequence of this external force on a cloud beyond the nuclear cluster is that the Roche density approaches zero. The reason for such a drop in Roche density is that the differential force across a cloud is not significant in the radial direction as long as the rotation curve is flat or rising. Thus, the acceleration across the radial direction becomes insignificant. This situation is different for a cloud embedded in a potential of a point mass where the rotation curve drops rapidly as a function of distance from $\operatorname{Sgr} \mathrm{A}^{*}$. This result is different than what has generally been assumed that the gas density must be greater than the Roche density. It is conceivable that prominent young star clusters such as the Arches and Quituplet clusters benefited from the contribution of the nuclear cluster in compressing the gas cloud, thus the cloud becomes gravitationally unstable. If the potential is dominated by the Dark matter in the early universe, then star formation may be induced in a cloud as long as clouds of gas do not follow a point mass potential.

\section{References}

Chen, X., Amaro-Seoane, P., \& Cuadra, J. 2016, ApJ, 819, 138

Geballe, T. R., Najarro, F., Rigaut, F., \& Roy, J.-R. 2006, ApJ, 652, 370

Jog, C. J. 2013, MNRAS, 434, L56

Lu, J. R., Ghez, A. M., Hornstein, S. D., et al. 2009, ApJ, 690, 1463

Paumard, T., Genzel, R., Martins, F., et al. 2006, ApJ, 643, 1011

Sanchez-Bermudez, J., Alberdi, A., Schödel, R., et al. 2014, A\& AP, 572, L1

Stolte, A., Brandner, W., Grebel, E. K., Lenzen, R., \& Lagrange, A.-M. 2005, ApJ, 628, L113

Tsuboi, M., Kitamura, Y., Miyoshi, M., et al. 2016, PASJ, 68, L7

Wardle, M. \& Yusef-Zadeh, F. 2008, ApJ, 683, L37

Wardle, M. \& Yusef-Zadeh, F. 2014, ApJ, 787, L14

Yusef-Zadeh, F., Royster, M., Wardle, M., et al. 2013, ApJ, 767, L32

Yusef-Zadeh, F., Roberts, D. A., \& Wardle, M., et al. 2015b, ApJ, 801, L26

Yusef-Zadeh, F., Wardle, M., Sewilo, M., et al. 2015a, ApJ, 808, 97 